\title{
Manufacture of Tapered Fibers and FBG Writing
}

\author{
C. C. Moura ${ }^{1}$, V. Oliveira ${ }^{1}$, C. R. Biazoli ${ }^{2}$, C. M. B. Cordeiro ${ }^{2}$, H. J. Kalinowski ${ }^{3}$ \\ ${ }^{I}$ Federal University of Technology - Paraná, 80230-901 Curitiba - Brazil, camilacmwill@gmail.com \\ ${ }^{2}$ University of Campinas, Campinas - Brazil, cmbc@ifi.unicamp.br \\ ${ }^{3}$ Universidade Federal Fluminense, 24210-240Niterói-Brazil, hjkalin@gmail.com
}

\begin{abstract}
This article discusses procedures for production of tapered fibers in different diameters (tapers) and Bragg gratings writing (tFBG) in such fibers. The reflection spectra in different tFBGs are compared with a FBG written in standard single-mode fiber (SMF28). The gratings were characterized in terms of their thermal sensitivity. Values reasonably close to $11 \mathrm{pm} /{ }^{\circ} \mathrm{C}$ were expected and were obtained in the different gratings in tapers, confirming that the thermo-optical coefficient is predominant in relation to thermal expansion in gratings written in tapered fibers.
\end{abstract}

Index Terms-Tapered fibers, fiber Bragg gratings, thermal sensitivity.

\section{INTRODUCTION}

Sensors based on Bragg gratings in optical fibers (FBG) [1] present small size, signal encoded in wavelength, immunity to electrical interference and electromagnetic fields, they are also inert in the presence of a variety of chemical agents. Such characteristics make these sensors attractive for gas industries, petrochemical, steel and others [2]. The production of tapered fibers expands the field of applications due to the smaller diameter that enhances the interaction through the evanescent field. Applications in the chemical and biological subjects have been previously demonstrated [3], [4], [5], [6]. Bragg gratings written in tapered fibers (tFBG) can be obtained with arbitrary profiles [7] in the thinning region, allowing optimize the interaction with some sensor agent in the medium outside.

This paper presents results of fiber production of tFBG to tens of micrometers in diameter, as well as writing and characterization for thermal sensitivity in gratings produced in such fibers.

\section{BIBLIOGRAPHIC REVIEW}

\section{A. Bragg Wavelength}

For a uniform Bragg grating, the wavelength center of the reflection spectrum occurs close to the Bragg wavelength, $\lambda_{B}$, of the grating, which is given by [2], as shows the equation 1 :

$$
\lambda_{\mathrm{B}}=2 n_{\mathrm{eff}} \Lambda
$$

where $n_{\text {eff }}$ is the effective refractive index of the propagated mode and $\Lambda$ is the spatial periodicity of the grating. For normal incidence of UV beam on the phase mask and fiber parallel mask, $\lambda_{\mathrm{B}}$ is 
determined by the phase mask period $\left(\Lambda_{\mathrm{PM}}\right)$ and by effective refractive index of the fiber, by means of the following relation [8]:

$$
\lambda_{\mathrm{B}}=n_{\mathrm{eff}} \Lambda_{\mathrm{PM}}
$$

where $\Lambda_{\mathrm{PM}}=\Lambda / 2$. It has already been observed that the reduction of the fiber diameters optimizes the grating writing process [5].

\section{B. Thermal sensitivity}

Any perturbation that changes the effective refractive index of propagation mode or the modulation pitch induces a shift in the Bragg wavelength in FBG, thus, one can measure a determined parameter by means of the spectral displacement of the peak reflected by the Bragg gratings. This characteristic makes the FBG an intrinsic sensor for temperature and strains these disturbances change the refraction and / or the pitch of the grating [8].

In the application of FBG as a temperature transducer, as shown in equation 3, a temperature variation, $\Delta \mathrm{T}$, entails a wavelength deviation $\Delta \lambda_{\mathrm{BT}}$ expressed by [8]:

$$
\Delta \lambda_{\mathrm{BT}}=\lambda_{\mathrm{B}}(\alpha+\xi) \Delta T
$$

where $\alpha$ is the coefficient of thermal expansion of the fiber material and $\xi$ is the thermo-optical coefficient of the fiber. The dominant term is the last, the first being significant only at temperatures above $800{ }^{\circ} \mathrm{C}$. For the standard silica fiber, the sensitivity of the FBG wavelength to the temperature, at $1550 \mathrm{~nm}$, is in the range of $8-14 \mathrm{pm} /{ }^{\circ} \mathrm{C}$, depending on the recording conditions.

\section{METHODOLOGY}

\section{A. Tapered fiber fabrication}

The process of manufacturing microfibers is analogous to conventional fiber manufacturing process and takes place from of the production of tapers, that is to say, tuned fibers. As with fibers, a preform, which in this case will be a standard SMF fiber (SMF28), will be heated allowing to draw the (reduced diameter) fiber. In order to fine-tune it to the desired diameter and length, the fiber is, simultaneously, heated and tensioned. Heating it to the point of reducing viscosity and, consequently, by relaxing the perpendicular stresses and increasing the shear stresses in the direction of fiber axis, the pulling will stretch it and, from mass conservation, it will increase the length and decrease its diameter. The temperature of heater for taper production was $1200{ }^{\circ} \mathrm{C}$ and the time production was ten minutes at that temperature. The traction speed was $0.03 \mathrm{~mm} / \mathrm{s}$. The tapered transition was controlled by the traction speed. The length of the tapered region in fiber is inversely proportional to the taper diameter, also exist some correlation with the shape of the transition, which in this article is conical. 


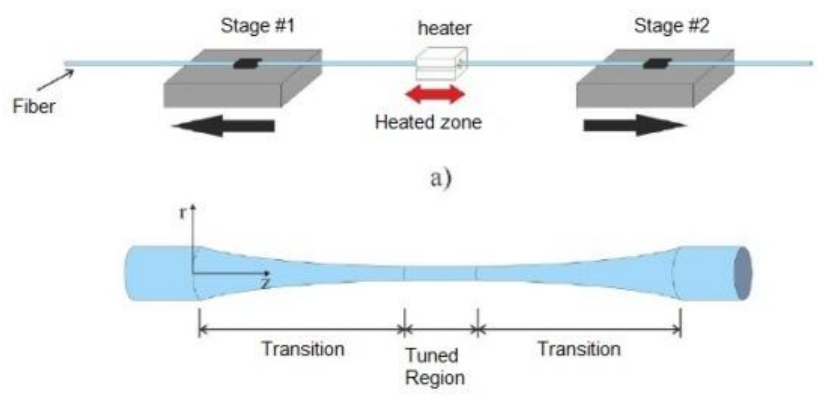

b)

Fig. 1 - Process for manufacturing fine-tapered fibers.

A well-recognized - and simple - mathematical model that describes the dynamics of this process had been given by T. Birks [9]. In this model, the author considered the following configuration: a fiber fixed to two linear stages aligned with opposing trajectories and a heated region dependent on the elongation length of the fiber. Fig.(1a) illustrates such configuration. The obtained fibers have two regions: transition region and tuned region - depicted in Fig. (1b), where last region is also called microfiber and therefore, its final product. This model provides that, starting from the evolution of the heated region, it is possible to obtain several transitions.

\section{B. Writing Bragg Gratings}

The method used for the inscription of Bragg gratings in standard SMF28 fiber and in tapered optical fiber is the direct writing through a phase mask [10]. It was used as UV laser source Coherent Xantos XS 500 - $193 \mathrm{~nm}$ and the broadband light source ALS-10-M Amonics® ASE (amplified spontaneous emission) module with output power $10 \mathrm{dBm}$ and operation wavelength $1527 \mathrm{~nm}$ to $1566 \mathrm{~nm}$. The interrogation was performed by an optical spectrum analyzer (OSA) Yokogawa AQ6375, ASE and optical circulator, as setup shown in Fig. 2.

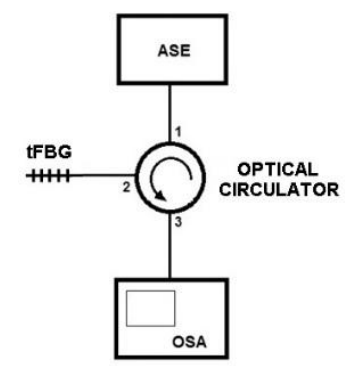

Fig. 2 - Optical circuit used to interrogate tapers with ASE, OSA and optical circulator.

The phase masks used, Ibsen, had pitches of $1073.50 \mathrm{~nm}$ and $1058.9 \mathrm{~nm}$, without chirp and are optimized to $193 \mathrm{~nm}$ illumination. All tFBGs and FBG have a length of $3 \mathrm{~mm}$ and were written using a repetition rate of $250 \mathrm{~Hz}$, energy of $2.5 \mathrm{~mJ}$ pulse, exposure time 2 minutes. The gratings were written in the region of smaller and constant diameter (tuned region in fig. 1b) in the tapered fiber. 
Techniques for increasing photosensitivity [11] on optical fibers were not applied in the preprocessing of the samples.

\section{Thermal sensitivity determination}

The tapered fibers, in the region of the FBGs, were heated using a Peltier module providing stabilized temperatures in the range of $0-100^{\circ} \mathrm{C}$ in steps of $10^{\circ} \mathrm{C}$ for tFBGs written with pitch $1073.50 \mathrm{~nm}$ and temperatures between $10-70^{\circ} \mathrm{C}$ in steps of $10^{\circ} \mathrm{C}$ for $\mathrm{tFBGs}$ written with pitch $1058.90 \mathrm{~nm}$; in both cases the temperature is set and adjusted through a digital controller unit. For efficient thermal coupling between the Peltier thermoelectric pellet and the sample fiber, a mineral oil was used at the interface. After stabilization of the temperature at each step a spectral acquisition was performed using the OSAs. The thermal sensitivities were determined the peak wavelength values $\left(\approx \lambda_{B}\right)$ of the spectra reflected by the FBGs and the corresponding temperatures. A second order polynomial fit was chosen to obtain the thermal coefficient of the function $\lambda_{B}=f(T)$, because it results the smallest error for this type of sensor [12].

\section{RESULTS}

Assuming that in the process of manufacturing the tapered fibers the proportions between the core and the cladding diameters are kept, that nominally determines the diameters of respectively, $125 \mu \mathrm{m}$ and $8.2 \mu \mathrm{m}$ [13]. TABLE 1 shows the values of the core diameters for the different tapered fibers.

TABLE 1- ESTIMATION FOR CORE DIAMETERS IN THE DIFFERENT TAPERED FIBERS

\begin{tabular}{cc}
\hline Fiber diameter $(\boldsymbol{\mu m})$ & Core diameter $(\boldsymbol{\mu m})$ \\
\hline 95 & 6.23 \\
\hline 80 & 5.25 \\
60 & 3.94 \\
40 & 2.62 \\
30 & 1.97 \\
\hline
\end{tabular}

An OEM XSZ-N107CCD optical microscope with a 40X objective lens was used to obtain images of FBG SM125, T80 and T40 tapers in the tuned region. Fig.3A) shows the optical fiber with regular diameter of $125 \mu \mathrm{m}$ and B) and C) shows the optical fibers with diameter of $80 \mu \mathrm{m}$ and $40 \mu \mathrm{m}$ respectively. 


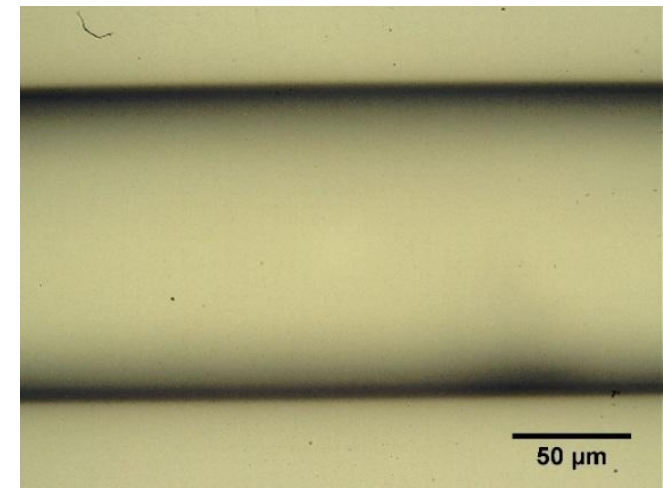

A)

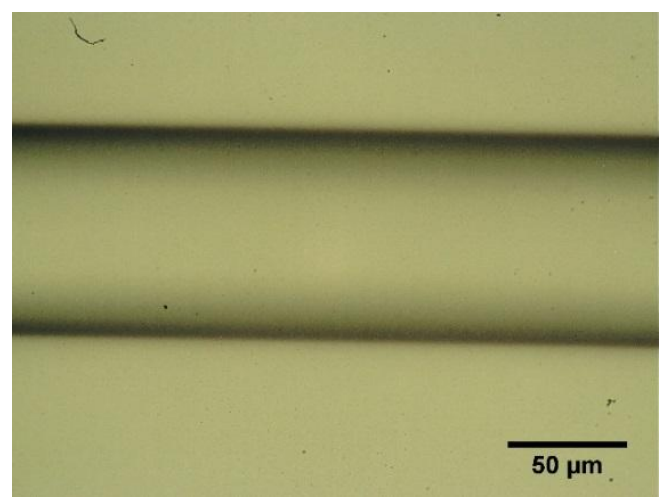

B)

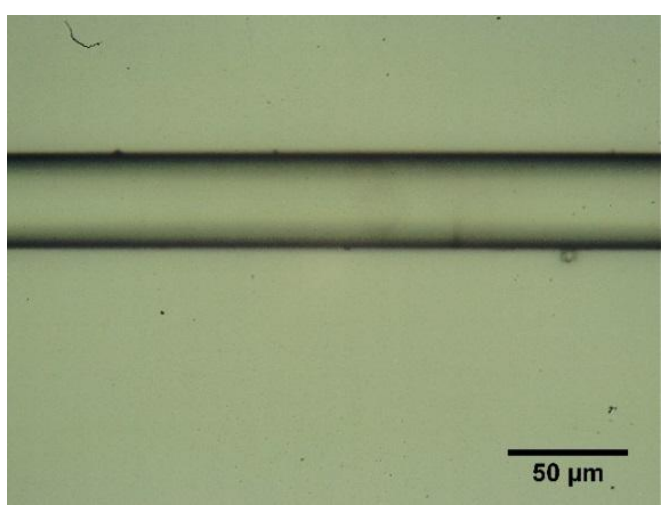

C)

Fig. 3 - Optical fibers diameters with A) $125 \mu \mathrm{m}$; B) $80 \mu \mathrm{m}$ and C) $40 \mu \mathrm{m}$ (see text for details).

Fig. 4 A) shows the spectra of the various gratings in the tapered fibers written with pitch 1073.50 $\mathrm{nm}$ and the fiber with the original diameter $(125 \mu \mathrm{m})$, at a temperature of $20^{\circ} \mathrm{C}$. There is an increase of $12 \mathrm{~dB}$ in amplitude of the signal reflected by the grating on the tapered fiber down to $60 \mu \mathrm{m}$ diameter (T60) compared to the grating in the fiber with nominal diameter (SMF28). Such an increase may be related to lower laser light attenuation through the thinner cladding during the inscription process and the different signal amplitudes obtained in different tapers may be due to the writing process, especially the critical condition for the beam alignment focusing on core of the optical fiber. For the fibers with 40 (T40) and 30 (T30) $\mu \mathrm{m}$ diameters, the amplitudes of the reflected signals were below that of the SMF28 grating, being $5.2 \mathrm{~dB}$, the largest difference presented, for a T30 tapered fiber.

The relatively low signal reflected by the T30 and T40 gratings seems to be related to the smaller fiber core used in the writing, which results in increased difficulty to reproduce the experimental conditions when aligning\& focusing the recording laser beam and also to a probable reduction of concentration of germanium in the core.

Fig. 4 B) shows the spectrum of tapered fibers A40 (40 $\mu \mathrm{m})$, A80 (80 $\mu \mathrm{m})$ e A95 (95 $\mu \mathrm{m})$ written with pitch $1058.9 \mathrm{~nm}$, at $20{ }^{\circ} \mathrm{C}$. There is a decrease of $6 \mathrm{~dB}$ in the amplitude of signal reflected between the tapered fiber A95 and A40, and there is also a shift in $\lambda_{B}$ of $10.89 \mathrm{~nm}$ from A40 to A95.The diameter with highest grating efficiency shown by results was T60 $\mu \mathrm{m}$ with $21 \mathrm{~dB}$ of signal amplitude. Tapers with diameters of $80 \mu \mathrm{m}$ have spectral amplitudes close to $10 \mathrm{~dB}$. The $40 \mu \mathrm{m}$ tapers 
presented spectra with reasonable equivalence in amplitude and spectral quality compatible with birefringence effect which is not the purpose of this paper.
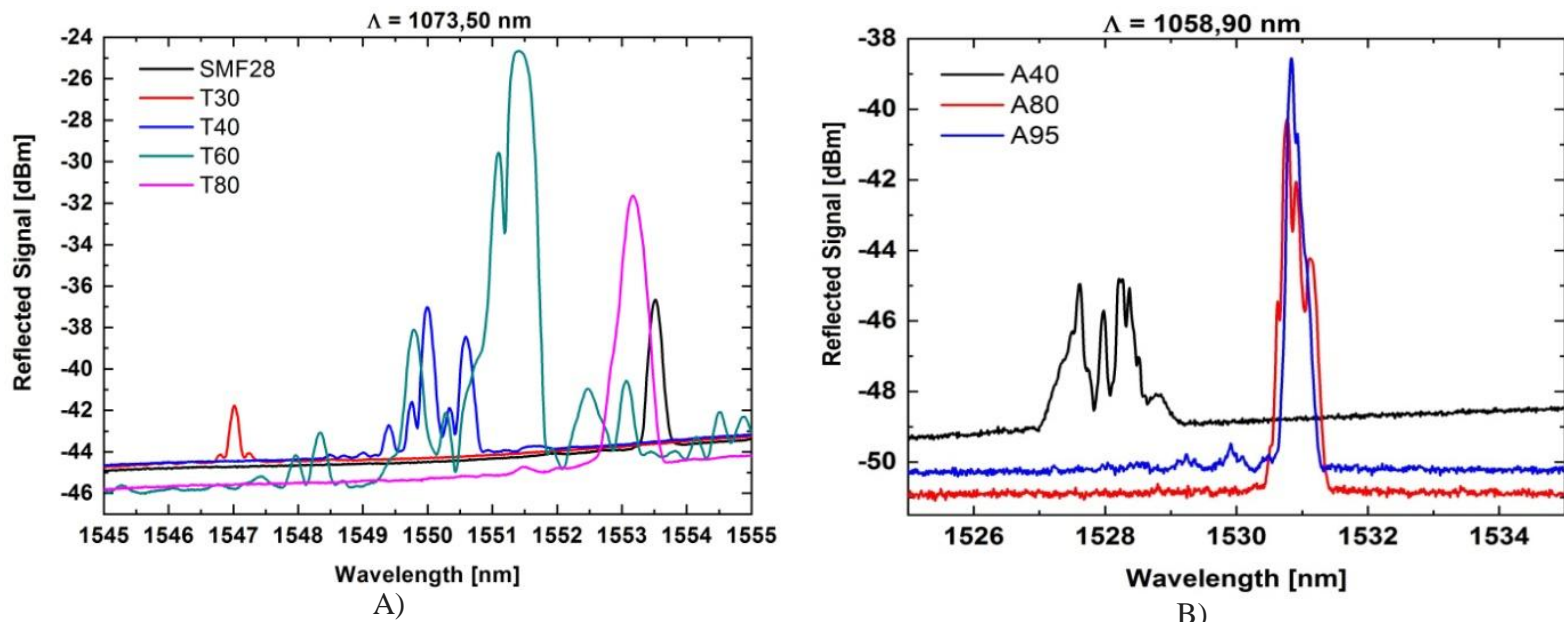

Fig. 4 - Signals reflected by Bragg gratings in fibers with different diameters for two different spatial pitches (see text for details).

Still in Fig. 4, it's seen a red shift in the Bragg wavelength, $\lambda_{\mathrm{B}}$, as a function of the fiber diameter. Such shift follows the effective refractive index behavior. Using equation (2) and the spectral positions shown in Fig. 4, we obtain the changes in $\mathrm{n}_{\text {eff }}$ as a function of the fiber diameter, as shown in the graphs on Fig. 3. The resulting values are $\mathrm{n}_{\text {eff }}=1.447$ for the fiber with its original diameter of 125 $\mu \mathrm{m}$ and 1.441 for the $30 \mu \mathrm{m}$ tapered fiber, from the samples inscribed with $1073.50 \mathrm{~nm}$ phase mask, whereas a slightly different set is obtained from the samples produced with the other phase-mask (Fig $5 \mathrm{~B}$ ) is shown in TABLE 2, where the refractive index values are similar and the variations are parameters dependent of the FBG recording process that is not fully controlled.

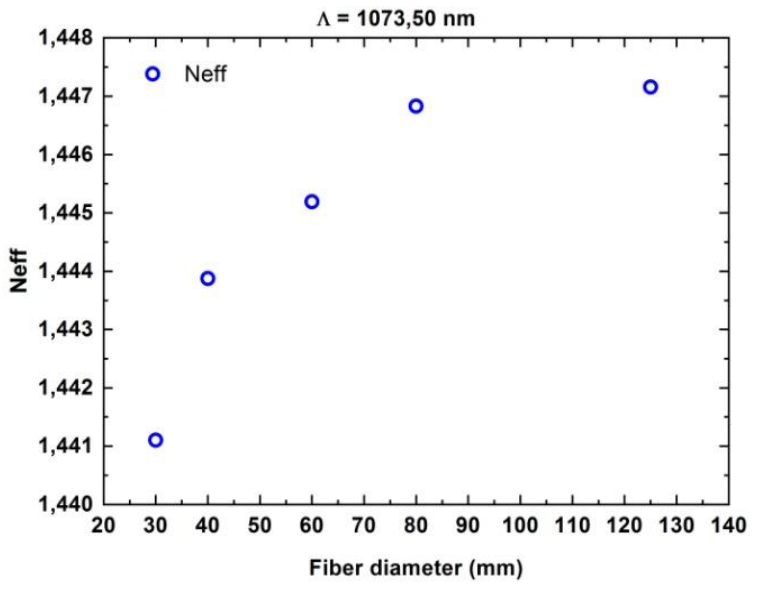

A)

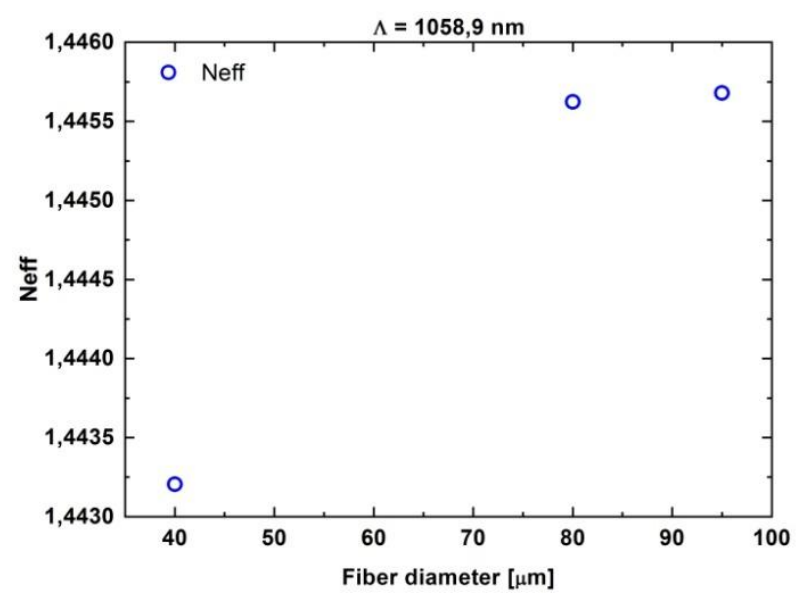

B)

Fig. 5-Evolution of effective refractive index $\left(\mathrm{n}_{\mathrm{eff}}\right)$ in two sets ofproduced gratings as a function of the final fiber diameter.

TABLE 2- EFFECTIVE REFRACTIVE INDEX FOR DIFFERENT TAPERED FIBERS

\begin{tabular}{cc}
\hline Grating & Effective refractive index $\left(\mathbf{n}_{\text {eff }}\right)$ \\
\hline T40 & 1.4438 \\
$\mathrm{~A} 40$ & 1.4432 \\
$\mathrm{~T} 80$ & 1.4468 \\
$\mathrm{~A} 80$ & 1.4456 \\
\hline
\end{tabular}


Fig.6A) shows the thermal sensitivity results for FBG written on the SMF28 fiber with original diameter and three tFBGs recorded on fine tapered fibers, T30, T40 and T60. Fig. 6B) shows thermal sensitivity results for three tFBGs recorded on fine tapered fibers A40, A80 and A95.

In TABLE3are shown the thermal sensitivities obtained for T30, T40, A40, T60, A80, A95tFBGs and FBG SMF28. The mean sensitivity of the set is $11.59 \mathrm{pm} /{ }^{\circ} \mathrm{C}$ and the largest deviation is $+15.53 \%$, presented by the A95 sample.

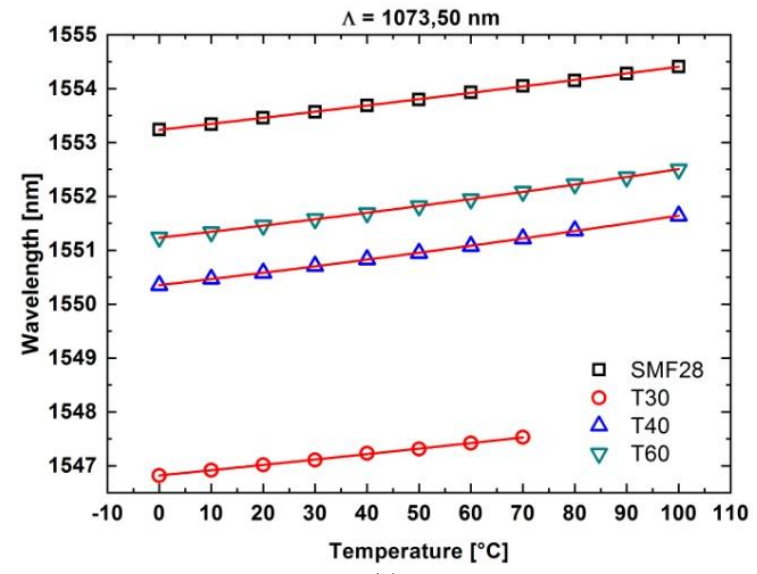

A)

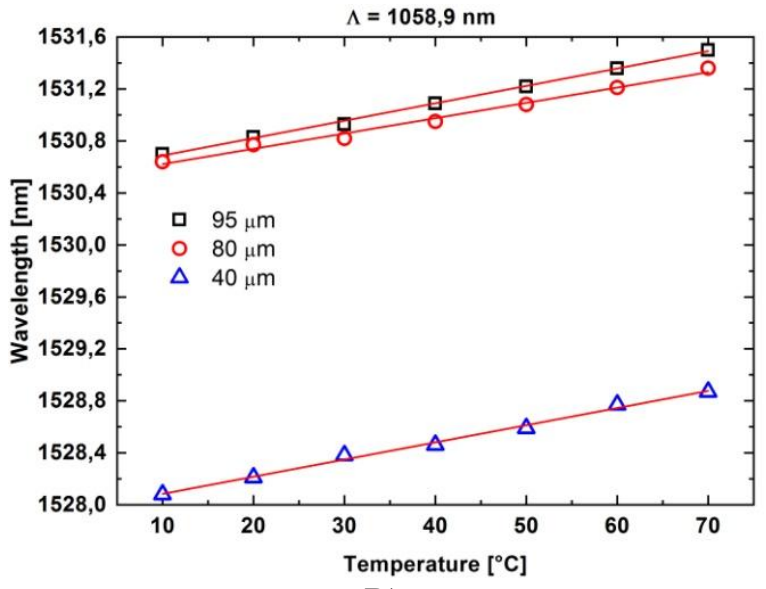

B)

Fig. 6 - Thermal sensitivities in FBG in standard and tapered fibers, for two recording pitches.

$\mathrm{T} 30$ was characterized only up to $70^{\circ} \mathrm{C}$ due to the difficulty of observing the response due to the small signal amplitude reflected in higher temperatures. The interaction of the evanescent field and the change in the refractive index of the oil used at the interface between the fiber and the thermo electric Peltier pellet could justify the amplitude drop in the reflected signal [14], but such a detailed analysis is not the focus of this job.

TABLE 3- THERMAL SENSITIVITY IN DIFFERENT BRAGG GRATINGS

\begin{tabular}{ccc}
\hline Grating & Thermal sensitivity $\left(\mathbf{p m} /{ }^{\mathbf{C}} \mathbf{C}\right)$ & Signal amplitude $(\mathbf{d B})$ \\
\hline SMF28 & 11.06 & 7.0 \\
T30 & 09.65 & 2.0 \\
T40 & 11.23 & 7.0 \\
A40 & 13.21 & 4.0 \\
T60 & 10.80 & 21.0 \\
A80 & 11.79 & 10.0 \\
A95 & 13.39 & 11.0 \\
Mean & 11.59 & 9.0 \\
\hline
\end{tabular}

\section{CONCLUSION}

The production of fine-tuned fibers and the writing of the Bragg grating in these fibers greatly expand the possibilities of applications for this type of fiber optic sensor. It is observed a reduction in the effective refractive index $\left(\mathrm{n}_{\text {eff }}\right)$ in the obtained Bragg gratings, caused by the reduction in the diameter of the used fibers. In terms of thermal sensitivity, it was not observed a significant change for the produced gratings with different diameters. This is consistent with the thermal sensitivity having a greater dependence on thermo-optical coefficient of the silica as compared to the dependence 
Journal of Microwaves, Optoelectronics and Electromagnetic Applications, Vol. 17, No. 4, December 2018

DOI: http://dx.doi.org/10.1590/2179-10742018v17i41549

with the thermal expansion of the fiber in the tapered zone.

\section{ACKNOWLEDGMENT}

This study was financed in part by the Coordenação de Aperfeiçoamento de Pessoal de Nível Superior - Brasil (CAPES) - Finance Code 001, FINEP, CNPQ and the Araucaria Foundation.

\section{REFERENCES}

[1] K.O. Hill, Y. Fujii, D. C. Johnson, B. S. Kawasaki, "Photosensitivity in optical fiber waveguides: application to reflection fiber fabrication, “Applied Physics Letters, vol. 32, issue 10, 1978.

[2] K.O. Hill, G. Meltz, "Fiber Bragg grating technology fundamentals and overview," Journal of Lightwave and Technology, vol. 15, issue 8, p.1263-1276, 1997.

[3] L. Zhang, J. Lou and L. Tong, "Micro/Nanofiber Optical Sensors," Photonic Sensors (2011) Vol. 1, No. 1: 31-42.

[4] X. Liu, T. Wang, Y. Wu, Y. Gong and Y.J. Rao, "Dual-Parameter Sensor Based on Tapered FBG Combined With Microfiber Cavity," IEEE Photonic Technology Letters, Vol. 26, No. 8, 2014.

[5] A. S. Paterno, V. Oliveira and H. J. Kalinowski, “Automated Photosensitive Enhancement in Optical Fiber Tapers,” J. Microwaves, Optoeletronics and Eletromag. applications, vol. 10, No. 1, June 2011.

[6] J. L. Kou, M. Ding, J. Feng, Y. Q. Lu, F. Xu and G. Brambilla. "Microfiber based Bragg gratings for sensing applications: a review," Sensors, vol.12, 2012.

[7] A. Felipe, G. Espindola, H.J. Kalinowski, J. A.S. Lima and A.S. Paterno, "Stepwise fabrication of arbitrary fiber optic tapers," Optics Express, vol. 20. no. 18, 19893, 2012.

[8] A. D. Kersey, M. A. Davis, H. J. Patrick, M. Leblanc and K. P. Koo, "Fiber grating sensors,” Journal of Lightwave Technology, vol. 15, issue 8, p. 1442-1463, 1997.

[9] T. A. Birks, Y. W. Li, “The shape of fiber tapers,” Lightwave Technology, Journal of, v. 10, n. 4, p. 432-438, 1992.

[10] A. Othonos and X. Lee, "Novel and Improved Methods of Writing Bragg Gratings with Phase Masks," Photonics Technology Letters, vol. 7, issue 10, 1995.

[11] F. Bilodeau, B. Malo, J. Albert, D. C. Johnson, K. O. Hill, Y. Hibino, M. Abe, and M. Kawachi, "Photosensitization of optical fiber and silica on silicon/silica waveguides," Opt. Lett., vol. 18, no. 12, pp. 953-955,1993.

[12] V. Oliveira, "Redes de Bragg para medições em altas temperaturas, “Tese de doutorado, Universidade tecnológica Federal do Paraná, 2012 .

[13] Catalog Corning, access:http://ece466.groups.et.byu.net/notes/smf28.pdf.

[14] R.C. Kamikawachi, I. Abe, H.J. Kalinowski, J.L. Fabris, J.F. Pinto, "Nonlinear temperature dependence of etched fiber Bragg gratings," IEEE Sensors Journal, vol 7, no. 8, 1358-1359, 2007. 